\title{
Index
}

Absolute purchasing power parity $105,156,165$

Aggregate demand 322,325 , $326,333,339,343,347,348$, 358,387

Akaike Information Criterion 393

Arbitrage 54, 58-61, 63, 64, 66-74, 76, 77, 202, 306

Asian consumer finance 284 Asymmetric responses 84, 92 Augmented Dickey-Fuller (ADF) $64,367,389,390$

Balassa-Samuelson model 124, 129-134, 138, 140-142, 166

Behavioral equilibrium exchange rate $102,128,159$

Big Bang 222, 230, 238
Bilateral swaps 196,197

Bond issuance $197,198,213$, $214,246,248,250,253,255$, $256,258,259,267,268$

Calibration 326,328

Canonical cointegrating regression 133,134

Capital account convertibility 56, 211

Capital controls $9,12,53,54$, 56-61, 63, 64, 66-68, 70, 74, $75,77,202,209,211,221,222$ De facto De jure index 9

Capital Flows 4, 8, 12, 56, 61, 78, 99, 104, 163, 202, 203, 220, 223, 236, 242, 247

Capital losses 191, 201, 207 


\section{Asia and China in the Global Economy}

Carry trade $240,245-250,253$, $258,259,268-270,273,274$

Center country $85,86,89,90$, 93

Chiang Mai Initiative (CMI)

195-197, 205

China factor $81,82,84-86,92$, 93

Chinese economy 194, 208

Chinese fiscal stimulus 323, 324, $334,336,347$

Cholesky ordering 393

Cointegration 124, 128, 130, 133-141, 143, 144, 382, 393, 398-400, 404

Competitive devaluation 81,82 , 84-86, 92

Consumer debt 284, 312

Covered interest parity 54,58 , $61,62,327$

Credit card crisis 284, 303, 309, $311,314,315$

Credit card lending boom-bust cycle 284,316

Credit card lending distress 284-286, 292, 293, 303, 305, 311

Credit card market 283-285, 294, 303, 313, 316

Credit information sharing and reporting 313

Credit reference agencies 284, 312

Credit scoring 284, 294

Crisis-related fiscal measures 336

Cross-border trade settlement 198
Currency denomination 245, 246, 248-251, 253, 255, 256, $262,268-270,273$

Currency misalignment 157,161 , $163,166,185$

Current account convertibility 221

DSGE model 324,326

Dynamic general equilibrium multicountry model 321,323

Economic growth $3,7,8,10,11$, $14,16,18,30,48,192$, 351-353, 356, 358, 361, 366, 381-383, 388

Elliott, Rothenberg and Stock

(ERS) Test 389

Emerging market economies 4-6, $10,13,16,19,26,28-30,35$, $39,41,42,47,58,68$

Equilibrium exchange rate 98, 100-108, 115, 116, 124, 128, 158-160, 163, 166, 177, 184, 185

Equilibrium real exchange rate

$120,123,128,144,146,157$, $160,162,164,184$

Euro-yen issues 226

Exchange rate $3,60,82,97,123$, $157,191,221,246,322,357$, 387

determination $82,92,114,148$ misalignment 100, 104, 107, 181 regime $8,60,93,133,141$, $159,210,322,323,334$, $339,340,343,347,348$ 
stability $3,4,6-9,18,21,24$, 26-31, 33, 36, 38-41, 46-49, 235

volatility $4,9,31-38,40-42$, 246,274

Export-led growth 356

Final Prediction Error 393

Financial crisis $54,56,63,156$, 185, 192, 193, 195-197, 203, 206, 240, 243, 246, 250, 268, $273,274,283,284,286,293$, $298,304,322,347,386,405$

Financial liberalization $8,30,38$, 39, 41, 209, 211, 212, 225-227, 284, 294, 313

Financial openness $4,8,18$, $27,29,30,38,39,41,46$, 48,49

Financial stability 214,215 , 283-285, 292, 313

Fiscal Investment and Loan

Program (FILP) 386

Fiscal multipliers 326

Fiscal package $323-325,336$, 343

Fiscal policy 19, 28, 206, 322, $328,332,347,348,366$, 381-383, 385-388, 395, 396, 404,405

Fiscal stimulus $319,321-324$,

$328,334,336,340,343$, 344-347

Fiscal stimulus package 321, $322,324,334,336$, 343-347
Fixed exchange rate regime 334 , $339,340,343,347$

Fixed nominal exchange rate 33 Foreign direct investment (FDI) $12,15,17,21,25,28-30,34$, $37,56,212,221,222,224$, 231,357

Foreign Exchange and Foreign

Trade Control Law 222, 223, 231

Foreign Exchange and Foreign

Trade Law 231

Foreign Exchange Council 224, $227,230,232,234$

Foreign Exchange Council Report 227,234

Foreign exchange reserves 56 , 191, 193, 201, 207, 220, 239, 240

Foreign Investment Law 223

Fundamental equilibrium exchange rate 102

GEM (Global Economy Model) 323

Global imbalances $156,157,323$, 348

Global trade and financial imbalances 322,348

Government Consumption 381, $388,395,396,398,404$

Government Investment 325, $381,386,389,395,397,398$, 404

Government Purchases 328, 381, 383-385, 387, 388, 


\section{2}

\section{Asia and China in the Global Economy}

391, 393-395, 397, 398, 403,404

Granger Block Exogeneity 393

Granger Causality $382,386,398$, 404

Grassman's law 221

Hanna-Quinn Information Criterion 393

Imbalances 156-158, 177, $178,292,311,322,323,348$ Impossible Trinity $6,7,48$ Inflation $3,4,6-8,10-12$, $18-20,22,23,25-29,31$, $32,34,35,37,48,56,141$, 201, 203, 207, 213, 246, $323,325,328,331-333$, $335,337-346$

Inflation volatility $6,7,10,11$, $19,20,22,23,25,27,28,31$, $32,34,35,37$

Information asymmetry 8,284 , $295,307,317$

International bond markets 246, $249,251,253,259,262$, 274

International bonds 245, 246, 248, 250-252, 255, 259, 270

International comparison program (ICP) 168

International coordination 348

International debt issues 273

International Financial Statistics

12,150
International monetary system 192, 204-207, 219-221, 242

International reserves $3,4,9,11$, $12,26,29,30,48,195$

Korean credit card crisis 311,315 Kwiatkowski, Phillips, Schmidt, And Shin (KPSS) Test 389

Law of one price $88,90,132,165$ Long-Term Economic Growth 353

Lost decade 383,404

Meta-analysis $97,98,100,101$, 105,106

Meta-regression 97, 106, 108, $110,112,117$

Misalignment 97, 98, 100-112, 114-117, 126, 128, 141, 146, 156-159, 161-164, 166-168, 170, 171, 173-176, 181, 184, 185

Model-based analysis 347

Monetary Independence 3, 6-9, 14, 16, 19, 21, 24, $26,29,31,33,36,38,41$, 46-49

New-Keynesian 323, 326

Non-deliverable forward market 54,59

Nontradables 124-132, 135, 137, 139, 140, 164, 324, 327, 330-332, 339 
Offshore renminbi market

Output Volatility 3-6, 10, 11, 18-20, 26-28, 30-32, 35, 38, $42,47,48$

Overvaluation $109,141,146$, 161,176

Panel data $159,284,303$, $309,352,354,356,367$, 379

Pearl and Yangtze River Delta 361

Penn World Table (PWT) 5, 11, 165

Phillip-Perron 367

Prais-Winsten procedure 175

Prediction error $168,173-175$, 393

Principal-agency problem 284 Productivity differentials 125 , $126,131,165$

Provincial data $355,357,362$, 365

Prudential and supervisory measures 284,317

Publication bias 101, 106, 114-116

Purchasing power parity (PPP) $100,102,103,105,128,158$, 165

Real demand rule 226, 229

Real exchange rates 39, 43, 46, $47,108,125,126,128,130$, $132,133,141,142,145,146$, $161,162,174$ misalignment 100,107 volatility $4,31-38,40-42$

Real income 11, 156, 174

Rebalancing 323

Relative purchasing power parity 158

Renminbi (RMB) 82, 95, 97-105, 110-112, 114-117, $124,126,136,142,146,155$, $156,160,191,192,361$ business $199,213,214$ denominated bonds 197, 198, 210,213 internationalisation 192-195, 197, 199, 200, 202-204, 207, 209-211, 215, 216

Revolvers 290, 297, 298, 309, 310,313

Roles of international currencies 194

Sampling uncertainty 173,175 Schwartz Information Criterion 389

Sequencing 192, 200, 210, 226

Serial Correlation $15,17,169$, 173-178

Simulations 333,336

Spillovers $\quad 323-326,339,378$

Terms-of-trade 11

Threshold auto-regression 54 , 67

Trade balance 88,99 , 180-184, 329, 335, $337,338,341$, 


\section{Asia and China in the Global Economy}

$342,344-346,354$, $360,361,378$

Trade openness 11, 14,

$16,18,19,22,23,32$,

$35,39,42-45,54,160,323$

Trade pattern $249,273,274$

Transactors 290,313

Trilemma $3,6-11,14,16,18-20$,

23, 27-31, 39-42, 47-49

Undervaluation 97,98 , 100-102, 104, 105, 108, $109,111,114,115,124$, $142,146,155,166,168$, $170,171,174,176-178$, $180,181,184$

Unit root nonstationary 131 Unit root tests $138,367,389$, 390

Vector Autoregression (VAR)

Model 126, 386
Vector Error Correction (VEC)

Model 382, 393, 398

Yen carry trade $245-247,268$, 270,273

Yen conversion 226, 229

Yen internationalization

220-222, 225-227,

231, 232, 234-236,

241,243

The Yen/Dollar Committee

225-227

Yen/Dollar Working Group 222, 225

Yen-denominated international bonds $245,248,251,259$, 270

The World Bank 167, 168, 171, 184,255

Zero Interest Rate Policy 385 